\title{
The Role of MKP-5 in Adipocyte- Macrophage Interactions during Obesity
}

\author{
Yuanhua Lu Jie Ma Jianan Zhao Zhuoyao Song Chao Zhou Xiu Liu \\ Wenjing Teng Wei Wang Qi Zhang Weiqun Yan Ping Jiao
}

School of Pharmaceutical Sciences, Jilin University, Changchun, China

\section{Keywords}

MKP-5 - Obesity $\cdot$ Macrophage $\cdot$ Adipocytes $\cdot$ Inflammation

\begin{abstract}
Objective: In obese individuals, chronic low-grade inflammation resulting from adipocytemacrophage interactions is a major cause of adipose tissue dysfunction and metabolic disease. This study investigated the role of MAP kinase phosphatase-5 (MKP-5) in obesity-induced inflammation during macrophage and adipocyte interactions. Methods: High-fat diet-induced obese mice were used to explore the role of MKP-5 in obesity-induced adipose tissue inflammation. Macrophage polarization was determined by inflammatory cytokine expression in MKP-5-overexpressed or -silenced Raw264.7 cells exposed to palmitate (PA) or M1/M2 macrophage inducers. To uncover the role of MKP-5 during macrophage-adipocyte interactions, a coculture system composed of differentiated 3T3-L1 and Raw264.7 cells was employed. MAPK inhibitors were used to investigate the involvement of MAPK signaling. $\boldsymbol{R e}$ sults: Increased MKP-5 expression was observed in adipose stromal vascular cells (SVCs) of obese mice. In Raw264.7 cells, MKP-5 promoted the switching of M1 macrophages to an M2 phenotype. Notably, MKP-5 reduced inflammation during the interaction of macrophages and adipocytes. MKP-5 overexpression in primary SVCs attenuated the expression of inflammatory mediators and increased the number of obesity-induced adipose tissue macrophages. MKP-5 suppressed PA-induced inflammation through the inactivation of P38, JNK, and ERK MAPKs. Conclusions: MKP-5 promotes macrophages to switch from the M1 to the M2 phenotype and is an inflammatory inhibitor involved in obesity-induced adipose tissue inflammation and PA-triggered macrophage inflammation via the P38, JNK, and ERK MAPK pathways. MKP-5 may be developed into a potential therapeutic target for obesity-related diseases, including type 2 diabetes mellitus and insulin resistance.


Lu et al.: MKP-5 Relieves PA or Obesity-Induced Inflammation

\section{Introduction}

Obesity is closely linked to serious conditions including elevated risks of insulin resistance (IR) and the closely related type 2 diabetes mellitus (T2DM) [1,2]. Research during the previous decade has linked chronic low-grade inflammation particularly to obesity-related T2DM [3, 4]. In obese adipose tissue, hypertrophic/hyperplastic adipocytes secrete several proinflammatory cytokines such as interleukin-6 (IL-6) and tumor necrosis factor- $\alpha$ (TNF- $\alpha$ ), as well as chemokines such as monocyte chemoattractant protein-1 (MCP-1), which drive macrophage infiltration, ultimately resulting in an adipose inflammatory response and IR [5-7]. As obesity progresses, the macrophages which infiltrate the adipose tissue themselves ultimately become the major producers of these chemokines and proinflammatory cytokines [5]. Of the two primary macrophage subtypes, M1 macrophages, which are generally regarded as being inflammatory, are major drivers of inflammation and IR in this context, whereas M2 macrophages are instead able to secrete anti-inflammatory cytokines and thus can help maintain metabolic homeostasis in adipose tissue [8]. Obesity drives adipose tissue macrophages to switch their phenotype from M2 to M1 [9-11]. Thus, paracrine and autocrine adipocyte-macrophage interactions can exacerbate the adipose tissue inflammatory response and IR development. In addition, free fatty acids (FFAs) released via lipolysis in adipose tissue are commonly elevated in obese individuals, and they can aggravate adipose tissue disorders, enhance inflammatory responses, and induce peripheral IR $[12,13]$. Inflammation is the primary driver of IR during obesity; however, the fundamental mechanism of obesity-related inflammation remains to be elucidated. Further identification of the signaling pathways that regulate adipocyte-macrophage interactions in adipose tissue is crucial, as it will provide novel insight that can help reduce obesity-related inflammation.

Mitogen-activated protein kinase phosphatases (MKPs) are enzymes which can specifically inactivate MAPKs by selectively dephosphorylating critical serine/threonine residues. MAPK proteins include P38, extracellular signal-regulated kinase (ERK), and c-Jun N-terminal kinase (JNK). There is growing evidence that MAPK signaling cascades represent critical inflammatory pathways that are intimately connected with adipose tissue inflammation and IR [14-16]. The function of MAPKs depends on the regulation of specific protein kinases and phosphatases, but the specific mechanisms governing the negative regulation of MAPKs in obesity-related T2DM are not fully clarified.

MAP kinase phosphatase-5 (MKP-5) is a JNK/P38-specific phosphatase important for innate and adaptive immunity [17]. Zhang et al. [18] demonstrated that MKP-5 knockout mice presented with substantially altered T-cell proliferation. The role of MKP-5 in inflammation has also been established in several tissues. For example, vitamin D reduces IL-6 production by inhibiting P38 activation via MKP-5 in normal prostate cells [19]. In aging diabetic mesangial cells, MKP-5 attenuates JNK activation and relieves the inflammatory response [20]. Recently, MPK-5 has been found to regulate brown adipocyte differentiation [21] and inflammation in white adipose tissue (WAT) via the P38 pathway [18], which indicates a potential role for MKP-5 in obesity-related metabolic disease. Hence, we assumed that MKP-5 suppressed the obesity-induced inflammatory response resulting from interactions between macrophages and adipocytes. The aim of our study was to elucidate whether MKP-5 affects macrophage-adipocyte interactions in response to obesity.

\section{Methods}

\section{Reagents}

High-glucose Dulbecco's modified Eagle's medium (DMEM) came from Corning (New York, NY, USA). Bovine serum was obtained from Gibco (California, USA), while Biological 
Lu et al.: MKP-5 Relieves PA or Obesity-Induced Inflammation

Industries (Kibbutz, Beit HaEmek, Israel) provided the fetal bovine serum (FBS). Recombinant mouse IL- 4 and IFN- $\gamma$ were obtained from Novus Biologicals (Centennial, CO, USA). The plasmids pHBAd-MCMV and pHBAd-BHGlox $\triangle \mathrm{E} 1,3$ Cre were from Hanbio Biotechnology (Shanghai, China), whereas pIRES2-EGFP-MKP-5 was purchased from Youbao Biotechnology (Guangzhou, China). TRIzol reagent, pcDNA3.1, Lipofectamine 2000, and RNAiMAX were obtained from Invitrogen (Carlsbad, CA, USA). The P38 inhibitor SB 203580 was from Calbiochem (San Diego, CA, USA). The JNK inhibitor SP 600125 and the ERK inhibitor U0126 were obtained from MedChemExpress (Monmouth Junction, NJ, USA).

LPS, insulin, dexamethasone, rosiglitazone, 3-isobutyl-1-methylxanthine, palmitate (PA), type I collagenase, fatty acid-free BSA, and ethyl alcohol (EtOH) were all from Sigma-Aldrich (St. Louis, MO, USA). Red blood cell lysate came from Beyotime (Shanghai, China). G418 was from Genview (Florida, USA). The PrimeScript RT reagent kit came from TaKaRa (Dalian, China). FastStart Universal SYBR Green Master (ROX) and protein inhibitor cocktail tablets were obtained from Roche (Basel, Switzerland). The Pierce ${ }^{\mathrm{TM}}$ BCA Protein Assay Kit was from Thermo Fisher Scientific (Waltham, MA, USA) and PVDF (polyvinylidene fluoride) membranes were from Millipore (Billerica, MA, USA). ECL luminescent liquid was purchased from Affinity (Ohio, USA). Cell Signaling Technologies (Danvers, MA, USA) was the source of antibodies to P38, phospho-P38 (pP38; T180/Y182), JNK, phospho-JNK (pJNK; T183/Y185), ERK1/2, and phospho-ERK1/2 (pERK1/2; T202/Y204). $\beta$-Actin and MKP-5 antibodies came from Abcam (Cambridge, UK). Anti-tubulin was from ABclonal Technology (Wuhan, China).

\section{Animal Model and Primary Stromal Vascular Cell and Adipocyte Isolation}

Male C57BL/6J mice (8 weeks old; Beijing Huafukang Bioscience, Beijing, China) were housed under standard conditions with free water access. The mice were fed either standard chow or a high-fat diet (HFD; $60 \%$ kcal from fat) for as many as 10 weeks, with weight being monitored once per week. They were fasted for $12 \mathrm{~h}$ prior to any procedure.

The mice were euthanized via $\mathrm{CO}_{2}$ inhalation. Epididymal fat pad adipocytes and stromal vascular cells (SVCs) were isolated as in a previous study [22]. Briefly, white fat pads were collected, rinsed, minced, and digested using $1 \mathrm{mg} / \mathrm{mL}$ collagenase I at $37^{\circ} \mathrm{C}$ for $45 \mathrm{~min}$ under gentle agitation. These digested fat pads then underwent $380-\mu \mathrm{m}$ mesh filtration, and cells were separated by centrifugation. Primary adipocytes were isolated by flotation, and they were used for the indicated downstream experiments. The stromal vascular fraction pellets were resuspended in red blood cell lysate and then underwent 106- $\mu \mathrm{m}$ mesh filtration. Isolated SVCs were either cultured with DMEM containing 10\% FBS for further study or used immediately.

\section{Cell Culture and Treatment}

Murine Raw264.7 macrophages were purchased from the American Type Culture Collection (ATCC) and were cultured using DMEM containing $10 \% \mathrm{FBS}$ at $37^{\circ} \mathrm{C}$ in a $5 \% \mathrm{CO}_{2}$ incubator. The pcDNA3.1-MKP5 plasmid or pcDNA3.1 empty plasmid was transfected into these cells using Lipofectamine 2000, and stable clones were isolated via selection in media containing G418 for 2-3 weeks. Briefly, the human MKP-5 coding sequence was obtained from the plasmid pIRES2-EGFP-MKP5 and inserted into pcDNA3.1 to produce the pcDNA3.1MKP5 plasmid. Raw264.7 macrophages stably expressing MKP-5 were referred to as Raw-MKP5 cells, while control macrophages were referred to as Raw-PC cells.

Raw264.7 cells underwent treatment with $20 \mathrm{ng} / \mathrm{mL}$ recombinant mouse IFN- $\gamma$ and 1 $\mu \mathrm{g} / \mathrm{mL}$ LPS for $6 \mathrm{~h}$ for M1 polarization, while $20 \mathrm{ng} / \mathrm{mL}$ recombinant mouse IL- 4 was used for $12 \mathrm{~h}$ to induce M2 polarization. SB $203580(10 \mu \mathrm{M})$, SP $600125(15 \mu \mathrm{M})$, or U0126 (5 $\mu \mathrm{M})$, which inhibit P38, JNK, and ERK, respectively, were applied to Raw264.7 cells $1 \mathrm{~h}$ after transfection with siRNA followed by PA $(500 \mu \mathrm{M})$ stimulation for $3 \mathrm{~h}$. PA was dissolved in EtOH to 
Lu et al.: MKP-5 Relieves PA or Obesity-Induced Inflammation

obtain a 50-mM stock and diluted using DMEM containing $0.5 \%$ fatty acid-free BSA to $500 \mu \mathrm{M}$ as a working solution.

Mouse 3T3-L1 preadipocytes came from the ATCC. For differentiation, preadipocytes were cultured using DMEM containing 10\% bovine serum, $1 \mu \mathrm{M}$ insulin, $1 \mu \mathrm{M}$ dexamethasone, $100 \mathrm{~nm}$ rosiglitazone, and $0.5 \mathrm{~mm}$ isobutylmethylxanthine for 3 days, followed by 7 days in DMEM containing 10\% FBS, $1 \mu \mathrm{M}$ insulin, and $100 \mathrm{nM}$ rosiglitazone. Mature 3T3-L1 adipocytes were used for coculture with macrophages.

\section{Macrophage-Adipocyte Coculture}

A contact coculture system was used to perform adipocyte-macrophage coculture assays as previously reported [23]. Briefly, Raw-MKP5 or Raw-PC cells $\left(1 \times 10^{5}\right.$ cells/well for a 6 -well plate) were seeded onto fully differentiated 3T3-L1 adipocytes for $24 \mathrm{~h}$, followed by PA or EtOH stimulation for $9 \mathrm{~h}$. The culture medium from this coculture system was then collected for measurement of inflammatory cytokine levels, and mRNA or protein was prepared. As a control, equal numbers of differentiated 3T3-L1 adipocytes and Raw-MKP5 or Raw-PC macrophages were also cultured individually, treated in the same manner, and mixed only after collection.

\section{Conditional Culture of Macrophages}

Differentiated 3T3-L1 adipocytes were treated using PA or EtOH for $12 \mathrm{~h}$ before collection of the adipocyte conditioned medium (CM). Raw-MKP5 or Raw-Pc macrophages were incubated in CM for $9 \mathrm{~h}$ and supernatants were harvested for ELISA.

\section{Construction and Preparation of an MKP-5-Expressing Adenovirus}

To construct an adenovirus expressing MKP-5, the human MKP-5 sequence was amplified by PCR using the plasmid pIRES2-EGFP-MKP5 as a template. MKP-5 was cloned into the shuttle plasmid pHBAd-MCMV and sequenced. The shuttle plasmid pHBAd-MCMV-MKP5 and the framework plasmid pHBAd-BHGlox $\Delta \mathrm{E} 1,3$ Cre were then cotransfected into 293A cells according to the manufacturer's instruction for virus production. Primary SVCs were seeded in 12-well plates at 60\% confluency, and infected using an adenovirus expressing MKP-5 (Ad-MKP5) or green fluorescent protein (Ad-GFP) $\left(1 \times 10^{9}\right.$ plaque-forming units $\left./ \mathrm{mL}\right)$ in DMEM with 10\% FBS for $6 \mathrm{~h}$, after which fresh DMEM containing 10\% FBS was added. After $48 \mathrm{~h}$, RNA or protein was prepared for further experiments.

Human 293A cells from the ATCC were cultured using DMEM containing 10\% FBS and penicillin/streptomycin.

\section{RNA Interference}

Small interfering RNA (siRNA) oligoribonucleotides targeting MKP-5 (siMKP5; Stealth RNAi $^{\mathrm{TM}}$ siRNA, Cat. \#8927232) and nonsense scramble siRNA (siSC; Stealth RNAi ${ }^{\mathrm{TM}}$ siRNA Negative Control, Cat. \#12935300) were synthesized by Thermo Fisher Scientific. These constructs (20 nM) were then transfected into Raw264.7 cells with Lipofectamine RNAiMAX. After $60 \mathrm{~h}$, macrophages were starved for $6 \mathrm{~h}$, pretreated with P38, JNK, or ERK inhibitor for $1 \mathrm{~h}$, then treated with PA for $3 \mathrm{~h}$, and then proteins were extracted for Western blotting.

\section{RNA Preparation and Quantitative Real-Time PCR}

Adherent cells were starved for 6-12 h prior to stimulation. Total RNA was extracted using TRIzol. Subsequently, cDNA was synthesized from $1 \mu \mathrm{g}$ total RNA with the PrimeScript RT reagent based on provided directions. Quantitative real-time PCR was performed with the FastStart Universal SYBR Green Master (ROX) reagent in a StepOnePlus system (Applied Biosystems, Carlsbad, CA, USA). The $2^{-\Delta \Delta C t}$ method was used to assess relative gene expression. The primers were as follows: 
- MKP-5: forward, 5'-TTAGACGACAGGGTAGTAGT-3', and reverse, 5'-GCTGGATGAGGGCATATA-3'

- IL-6: forward, 5'-CCACTTCACAAGTCGGAGGCTTA-3', and reverse, 5'-GCAAGTGCATCATCGTTGTTCATAC-3'

- IL-1 $\beta$ : forward, $5^{\prime}$-CCCTCCTGGCCAACGGCATG-3', and reverse, 5'-TCGGGGCAGCCTTGTCCCTT-3'

- Il-12: forward, 5'-CTCCAAGCCAAAGTCCTTAGAG-3', and reverse, 5'-AGGAGCTGTCATTAGGGACATC-3'

- MCP-1: forward, 5'-GGTCCCTGTCATGCTTCTGG-3', and reverse, 5'-TTGGGATCATCTTGCTGGTG-3'

- TNF- $\alpha$ : forward, $5^{\prime}$-CCCTCCTGGCCAACGGCATG-3', and reverse, 5'-TCGGGGCAGCCTTGTCCCTT-3'

- Arg-1: forward, 5'-CTCCAAGCCAAAGTCCTTAGAG-3', and reverse 5'-AGGAGCTGTCATTAGGGACATC-3'

- IL-10: forward, 5'-AATTCCCTGGGTGAGAAGCTGA-3', and reverse, 5'-CTCTTCACCTGCTCCACTGCC-3'

- TGM2: forward, 5'-AGGACATCAACCTGACCCTG-3', and reverse, $5^{\prime}$-CTTGATTTCGGGATTCTCCA-3'

- Mgl-1: forward, 5'-TGAGAAAGGCTTTAAGAACTGGG-3', and reverse, 5'-GACCACCTGTAGTGATGTGGG-3'

- $28 S$ rRNA: forward, 5'-TTCACCAAGCGTTGGATTGTT-3', and reverse, 5'-TGTCTGAACCTGCGGTTCCT-3'

\section{ELISAs}

Supernatant IL-6, TNF- $\alpha$, and MCP-1 levels were measured via DuoSet ELISA (R\&D Systems, Minneapolis, MN, USA) based on provided directions. Briefly, microtiter plates were precoated with capture antibodies specific to mouse IL-6, TNF- $\alpha$, or MCP-1 overnight. Following $1 \mathrm{~h}$ of blocking, cytokine standards and samples were incubated in these microtiter plates for $2 \mathrm{~h}$. After washing away unbound substances, detection antibodies were added and incubated for $2 \mathrm{~h}$. Streptavidin-HRP was then added for $20 \mathrm{~min}$ protected from light. Color development occurred following the addition of substrate solution (tetramethylbenzidine) and was proportional to the cytokine levels in the samples. Absorbance was assessed at 450 $\mathrm{nm}$ via Bio-Tek microplate reader (Bio-Tek, Winooski, VT, USA).

\section{Immunoblotting}

Tissues were homogenized and cells were scraped with lysis buffer (150 mM NaCl, $10 \mathrm{~mm}$ Tris pH 7.2, 5 mM EDTA, $0.1 \%$ SDS, $1 \%$ sodium deoxycholate, and $1 \%$ Triton X-100) containing protease inhibitors. The cell lysates were spun at $12,000 \mathrm{rpm}$ at $4{ }^{\circ} \mathrm{C}$ for $15 \mathrm{~min}$, and supernatants were collected for the next experiments. A Pierce ${ }^{\mathrm{TM}}$ BCA Protein Assay Kit was used to measure protein concentrations, with BSA as a standard. After SDS-PAGE electrophoresis, proteins were transferred onto PVDF membranes. 5\% nonfat milk in Tris-buffered saline with Tween 20 (20 mM Tris- $\mathrm{HCl}, 50 \mathrm{~mm} \mathrm{NaCl}$, and $0.1 \%$ Tween 20, pH 7.5) was used for blocking, and the blots were then incubated with primary antibodies followed by HRP-conjugated secondary antibodies. Finally, the blots were visualized using ECL luminescent liquid on a Tanon 5200 Multi FluorChem imaging system (Tanon, Shanghai, China).

\section{Statistical Analysis}

Two-tailed unpaired Student's $t$ tests and ANOVAs followed by LSD post hoc tests were used for all statistical comparisons. The data are given as means \pm standard error. $p<0.05$ was the threshold of significance. 


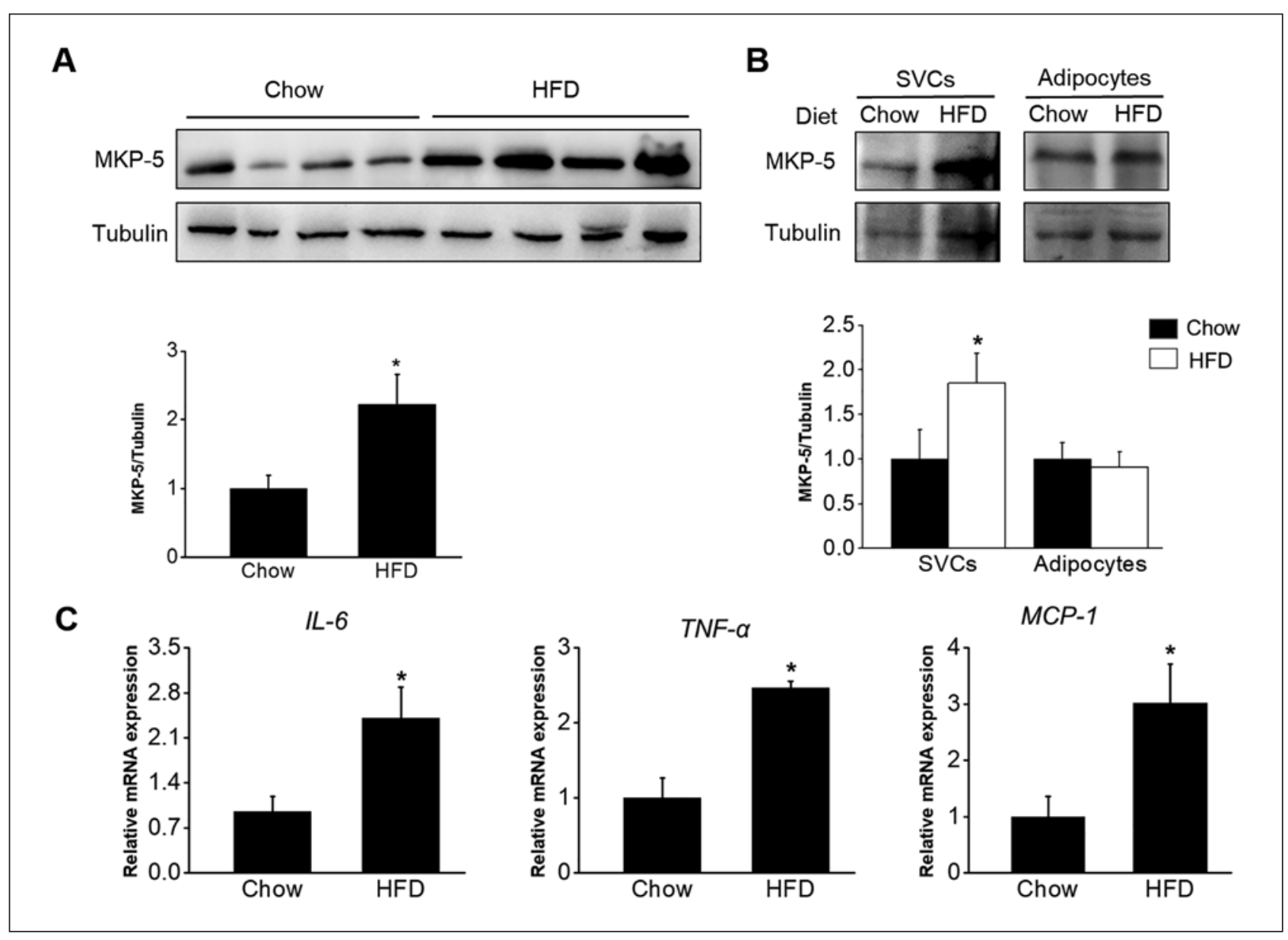

Fig. 1. MKP-5 is involved in obesity-induced inflammation. A White adipose tissue MKP-5 expression in highfat diet (HFD)- and chow-fed mice was measured via Western blotting. B Stromal vascular cells (SVCs) and adipocytes were isolated from HFD-fed $(n=5)$ and chow-fed mice $(n=8)$, and MKP-5 levels were assessed by Western blotting. C IL-6, TNF- $\alpha$, and MCP-1 expression was assessed via quantitative real-time PCR. The ImageJ software package was used for quantifying band densitometry, with tubulin used for normalization. ${ }^{*} p<0.05$.

\section{Results}

\section{MKP-5 Expression Is Modulated in Diet-Induced Obese Adipose Tissue}

Obesity is closely linked to both dysfunctional adipose tissue and chronic low-grade inflammation [24, 25]. When we assessed MKP-5 expression in murine WAT, we found it to be markedly increased in animals on an HFD (Fig. 1A). Furthermore, MKP-5 protein levels were elevated in SVCs but not in obese (HFD-fed) WAT adipocytes relative to controls (Fig. 1B), which indicated that the MKP-5 expressed in SVCs contributed to its elevated levels in WAT. IL- 6 and TNF- $\alpha$ levels were upregulated in obese WAT (Fig. 1C), as was expression of MCP-1, a chemokine which promotes macrophage infiltration (Fig. 1C). These changes in both MKP-5 and proinflammatory cytokine/chemokine levels indicated a possible role for MKP-5 in obesity-induced inflammation in WAT.

\section{MKP-5 Overexpression Promotes M1-to-M2 Macrophage Polarization}

Previous work suggests that adipose tissue macrophages are the key adipose tissue cells secreting proinflammatory molecules [26]. To confirm the relationship between MKP-5 and 
Lu et al.: MKP-5 Relieves PA or Obesity-Induced Inflammation

obesity-induced inflammation, murine Raw264.7 macrophages were treated with PA to trigger inflammation, and the expression levels of classic proinflammatory cytokines and MKP-5 were determined at several time points by quantitative real-time PCR. IL-6, IL-1 $\beta$, IL-12, and MKP-5 expression levels were upregulated after $3 \mathrm{~h}$ (Fig. 2A), after which IL-6 expression continued to rise. However, the expression of IL-1 $\beta$, IL-12, and MKP- 5 peaked at $3 \mathrm{~h}$, with lower expression at $6 \mathrm{~h}$ (Fig. 2A). The covariation of the expression of MKP-5 and proinflammatory cytokines suggested a potential role for MKP-5 in macrophage-mediated inflammatory responses upon PA stimulation. M1 macrophages are generally of a proinflammatory phenotype and promote IR development, while the alternatively activated M2 macrophages play an anti-inflammatory role and are important for the maintenance of WAT metabolic homeostasis $[9,27,28]$.

To study the role of MKP-5 in PA-triggered macrophage inflammation, an MKP-5-overexpressing Raw264.7 cell line was constructed, with increased MKP-5 expression confirmed by quantitative PCR and Western blotting (Fig. 2B). IL-6, IL-1 $\beta$, IL-12, TNF- $\alpha$, and MCP-1 levels were significantly increased upon stimulating these cells with IFN- $\gamma$ and LPS to promote M1 polarization, whereas the expression levels of these molecules were downregulated in Raw-MKP5 cells relative to Raw-PC cells with or without IFN- $\gamma$ and LPS stimulation (Fig. 2C). M2 markers (Arg-1, IL-10, and TGM2) were increased when Raw-PC and Raw-MKP5 cells were exposed to IL-4, used to induce M2 polarization. In contrast to the expression of M1 markers, Arg-1, IL-10, and TGM2 levels in Raw-MKP5 cells were significantly higher than in Raw-PC cells (Fig. 2D).

Consistent with previous reports [7, 8], PA promoted the expression of M1 markers in both Raw-PC and Raw-MKP5 cells, but the effect in Raw-MKP5 cells was weaker than that in Raw-PC cells (Fig. 2E), consistent with the results under IFN- $\gamma$ and LPS stimulation (Fig. 2C). PA could not affect Arg-1 and IL-10 expression in Raw-PC cells, but it clearly inhibited these molecules in Raw-MKP5 cells. Importantly, MKP-5 overexpressed in Raw264.7 cells promoted expression of the M2 markers (Fig. 2F). It is therefore conceivable that MKP-5 overexpression promotes macrophages to polarize from the M1 to the M2 phenotype.

\section{Reduction of MKP-5 Expression in Macrophages Triggers an M2-to-M1 Macrophage}

Switch upon PA Stimulation

To further validate that MKP-5 promotes M1 macrophages to polarize to the M2 phenotype, we reduced the expression of MKP-5 by transfecting an MKP-5-specific siRNA (siMKP5) into Raw264.7 cells, leading to decreased mRNA and protein levels of MKP-5 relative to cells transfected with scramble siRNA (siSC) (Fig. 3A, B). In line with the results from Raw-PC/-MKP5 cells, PA induced M1 marker upregulation, including IL-6, IL-1 $\beta$, and TNF- $\alpha$, and elevated expression of these markers was observed after MKP-5 knockdown (Fig. 3C). Levels of the M2 markers Arg-1 and IL-10 were decreased upon PA stimulation, and knockdown of MKP-5 further inhibited their expression (Fig. 3D). In general, MKP-5 silencing in macrophages promoted M1 polarization and exacerbated the PA-induced inflammatory response, providing further evidence for the inhibitory role of MKP-5 in obesity-triggered inflammation.

Fig. 2. MKP-5 overexpression in macrophages is involved in M1-to-M2 macrophage polarization. A $500 \mu \mathrm{M}$ palmitate (PA) was used to treat Raw264.7 cells, and then IL-6, IL-1 $\beta$, IL-12, and MKP-5 expression was detected at 1, 3, and 6 h by quantitative real-time (qRT)-PCR. B A stably transfected MKP-5 Raw264.7 cell line was constructed, and MKP-5 overexpression was assessed by qRT-PCR and Western blotting. C Raw-PC and Raw-MKP5 cells were stimulated with the indicated concentration of IFN- $\gamma$ and LPS for $6 \mathrm{~h}$, and M1 markers were measured by qRT-PCR. D Raw-PC and Raw-MKP5 cells were stimulated using IL-4 for $12 \mathrm{~h}$, and M2 markers were measured by qRT-PCR. E, F With $500 \mu \mathrm{M}$ PA for $6 \mathrm{~h}$, the expression levels of M1 (E) and M2 markers (F) in Raw-PC and Raw-MKP5 cells were detected by qRT-PCR. Results are means \pm SEM of triplicate experiments. EtOH, ethyl alcohol. ${ }^{*} p<0.05$.

(For figure see next page.) 
A
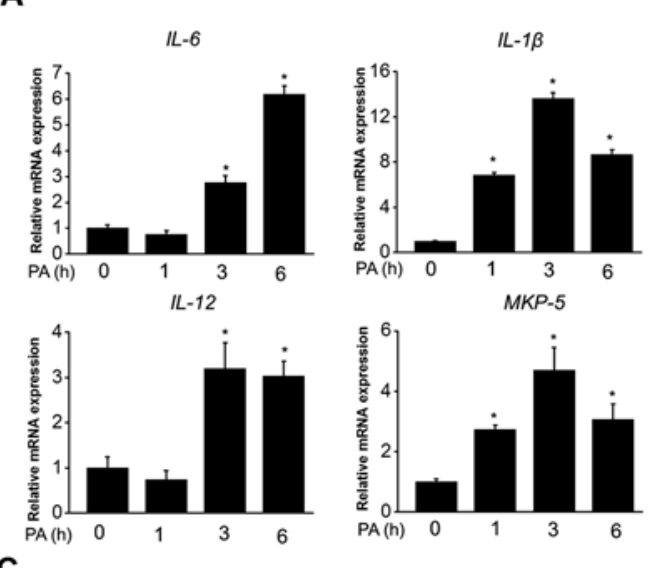

C
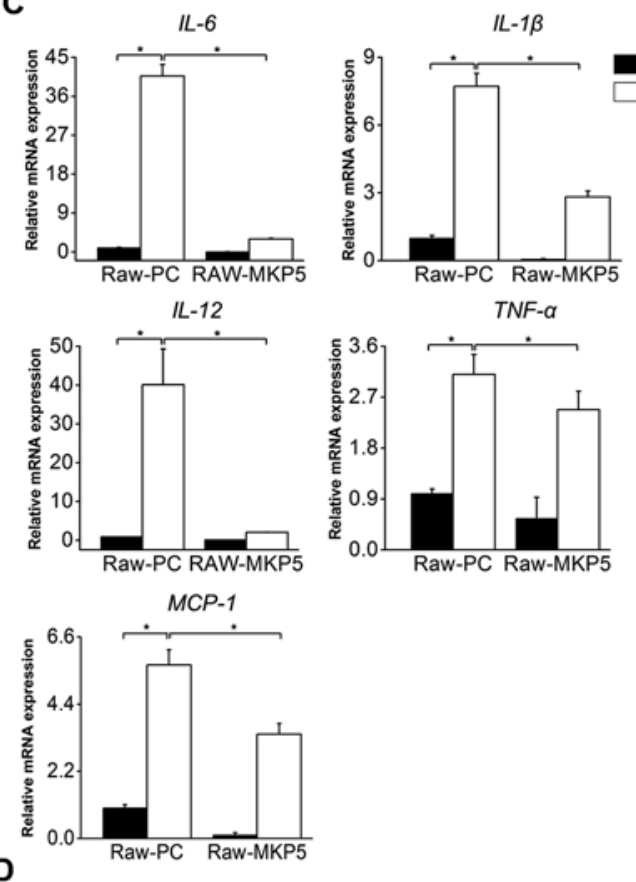

D
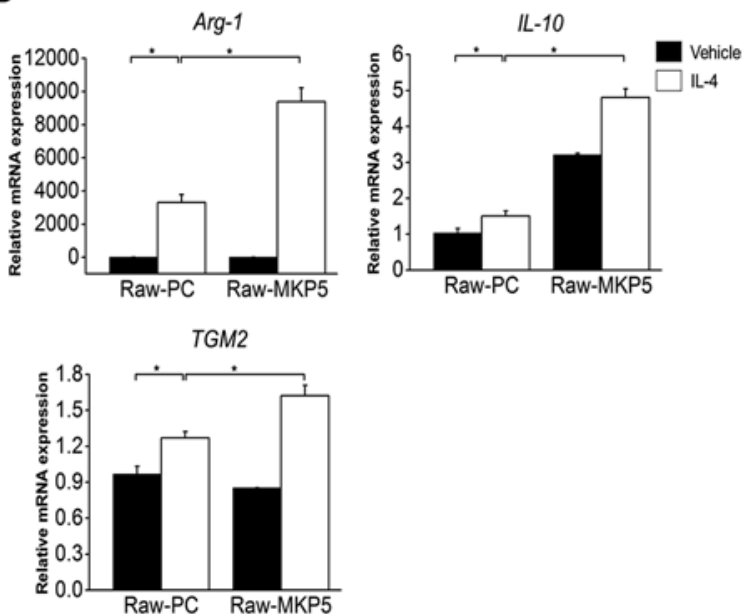

B

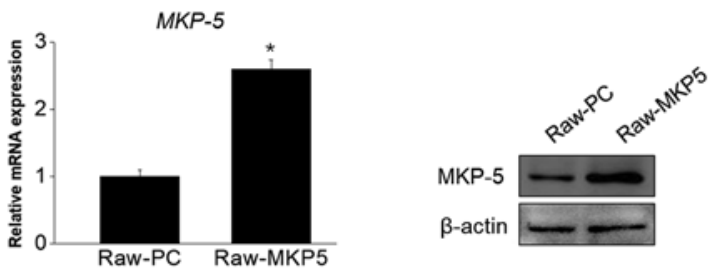

E
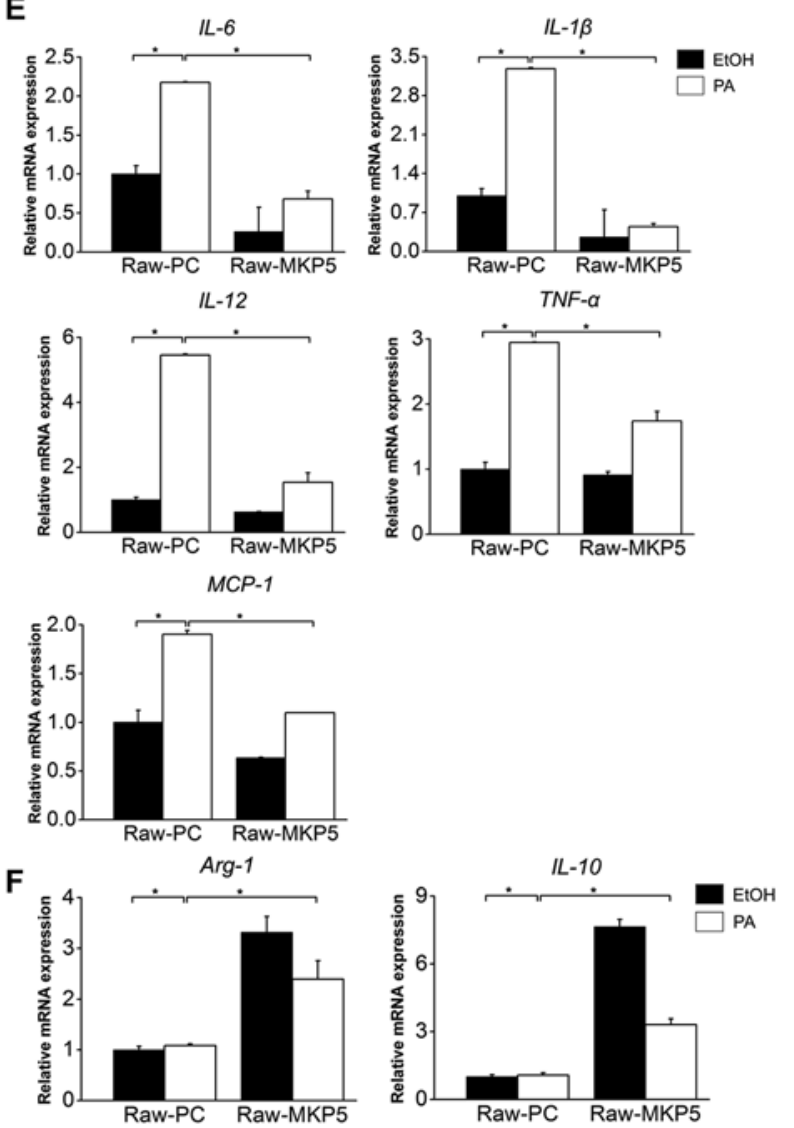


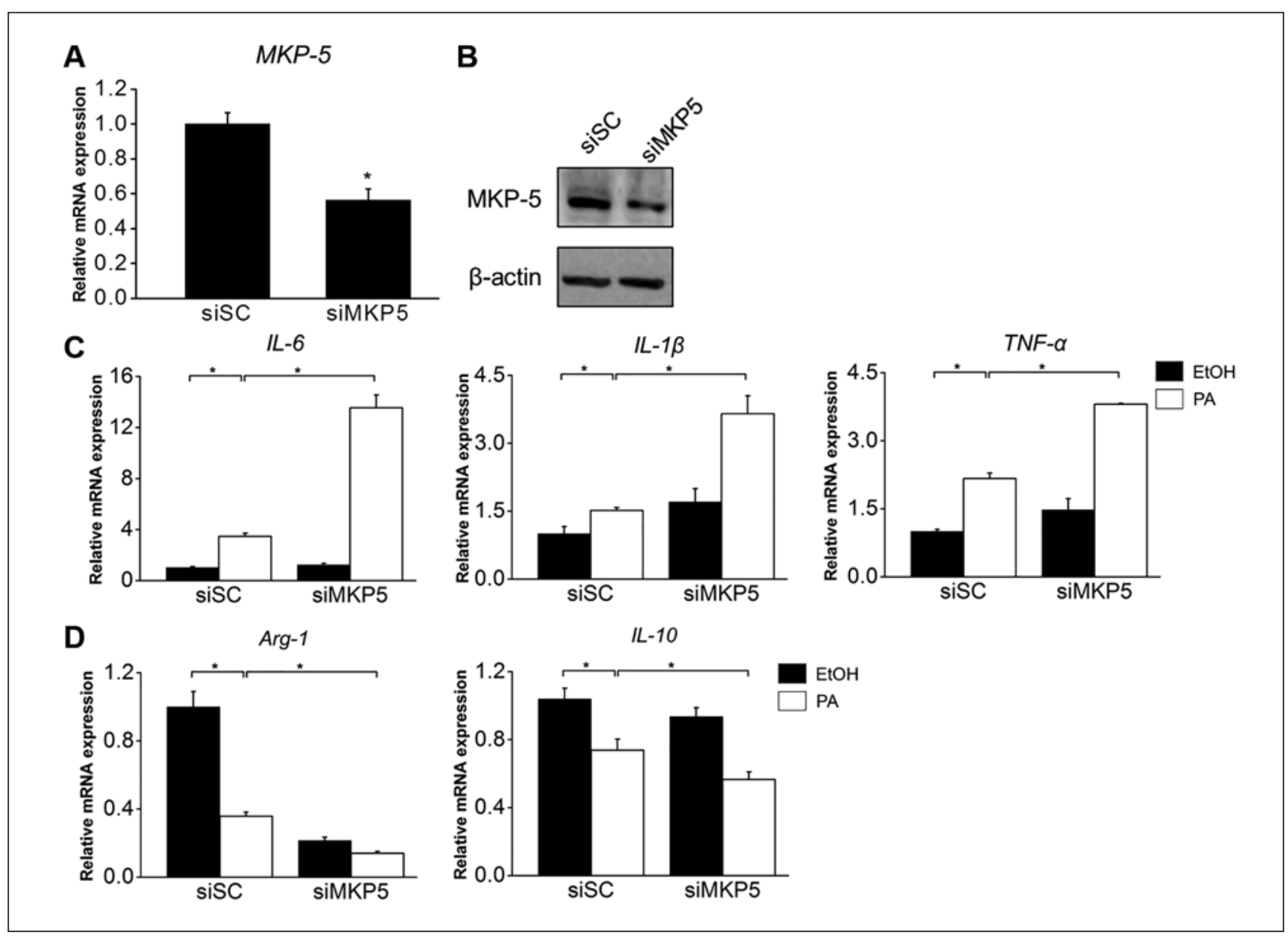

Fig. 3. Silencing MKP-5 in macrophages mediates M1 and M2 marker expression in response to palmitate (PA). A, B Raw264.7 cells were transfected using MKP-5-specific siRNA, and MKP-5 expression was assessed via quantitative real-time (qRT)-PCR (A) and Western blotting (B). C, D After transfection with MKP-5-specific siRNA, Raw264.7 cells were incubated with $500 \mu \mathrm{M}$ PA for $6 \mathrm{~h}$ (C) or $9 \mathrm{~h}$ (D). Relative expression of the M1 markers (C) and M2 markers (D) was then measured by qRT-PCR. Results are means \pm SEM of triplicate experiments. EtOH, ethyl alcohol. ${ }^{*} p<0.05$.

\section{MKP-5 Overexpression in SVCs Reduces Obesity-Induced Inflammation}

To further determine the importance of MKP-5 in obesity-induced inflammation in SVCs, obese mice were generated via HFD feeding, and SVCs from the WAT of these mice were isolated and infected with Ad-MKP5 or Ad-GFP. The mRNA and protein levels of MKP-5 in these obese SVCs were increased, consistent with the above results, and Ad-MKP5 infection significantly increased the expression of MKP-5 relative to cells infected with Ad-GFP (Fig. 4A, B). We first examined MCP-1 and macrophage-specific F4/80 expression in SVCs. Levels of MCP-1 and F4/80 were substantially elevated in obese WAT relative to their levels in lean WAT, while adenovirus-mediated MKP-5 overexpression downregulated this high expression, indicating that MKP-5 overexpression may inhibit obesity-induced macrophage infiltration (Fig. 4C). Consistent with this, the obesity-triggered increases in mRNA levels of IL-6 and TNF- $\alpha$ were decreased by MKP-5 overexpression in SVCs (Fig. 4C). In general, while in obesity the synchronous changes in expression of MKP-5 and proinflammatory cytokines contradicted the anti-inflammatory role of MKP-5 identified using Raw264.7 cells, the inhibitory role of MKP-5 in inflammation was further validated in SVCs. 


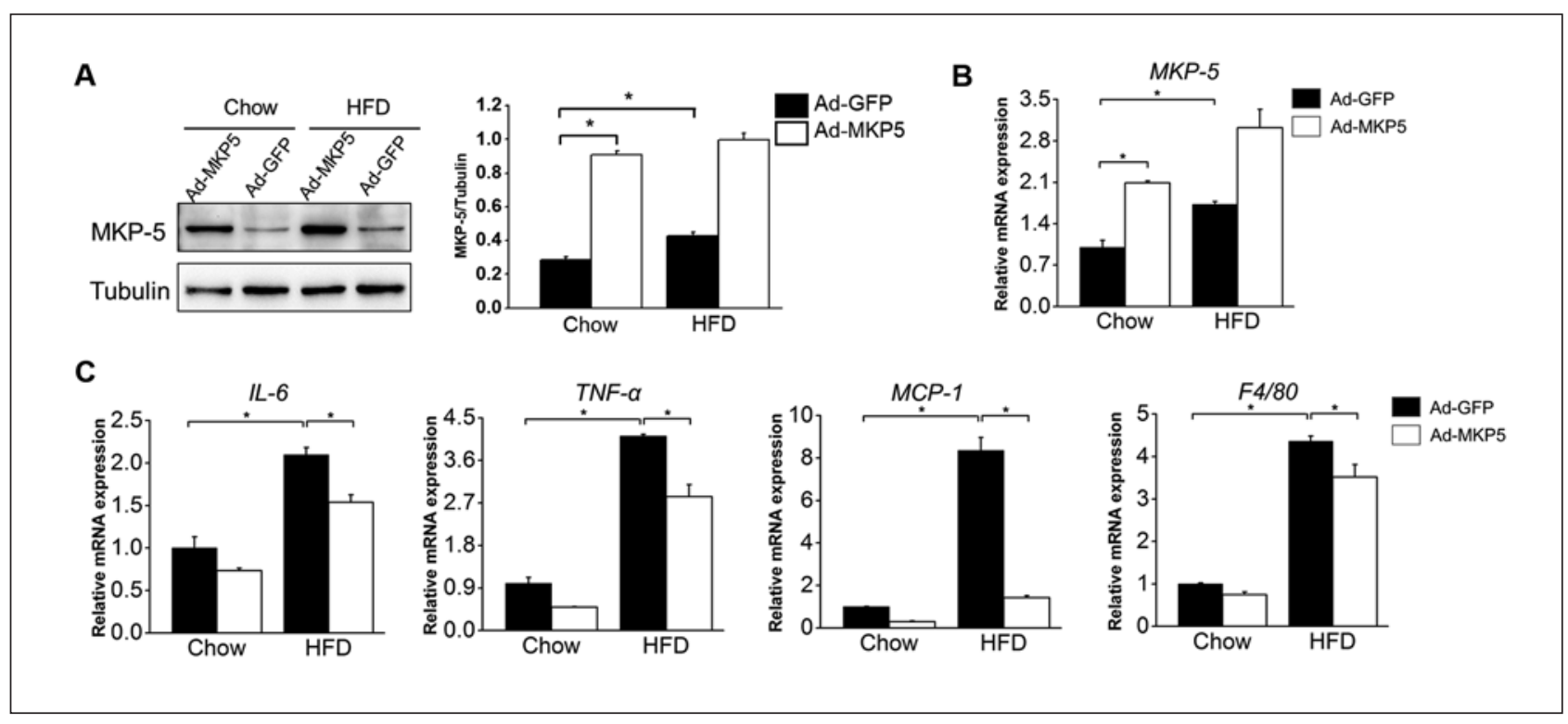

Fig. 4. MKP-5 can improve obesity-induced inflammation in stromal vascular cells (SVCs). SVCs were isolated from white adipose tissue of high-fat diet (HFD)- or chow-fed mice and then infected with an adenovirus expressing MKP-5 (Ad-MKP5) or green fluorescent protein (Ad-GFP). A Levels of MKP-5 were determined via Western blotting, and protein band intensity was quantified using ImageJ. Data are means \pm SEM of triplicate experiments normalized to tubulin. B mRNA levels of MKP-5 were detected by quantitative realtime (qRT)-PCR. C F4/80, IL-6, TNF- $\alpha$, and MCP-1 expression levels in SVCs were detected by qRT-PCR. Results are means \pm SEM of triplicate experiments. $* p<0.05$.

\section{MKP-5 Overexpression in Macrophages Alleviates Inflammation during}

Adipocyte-Macrophage Interaction in vitro

Macrophage-adipocyte crosstalk is a key contributor to obesity-triggered inflammatory response [29]. To explore the role of MKP-5 expression in macrophages during this process, macrophages were cocultured with adipocytes. A marked increase in IL-6 levels was observed upon coculture of these cells as compared to macrophages or adipocytes cultured alone (Fig. 5A, B). A significant increase in TNF- $\alpha$ secretion was also detected in the coculture samples (Fig. 5B). This effect was amplified upon PA exposure (Fig. 5A, B). Importantly, expression and secretion of proinflammatory cytokines were markedly suppressed during adipocyte-Raw-MKP5 cell coculture relative to the levels observed upon adipocyte-Raw-PC cell coculture, and PA stimulation did not alter this phenomenon (Fig. 5A, B). These results suggested that MKP-5 plays an anti-inflammatory role in the context of adipocyte-macrophage coculture. The mRNA and protein levels of the chemokine MCP-1 were also increased by adipocyte-macrophage coculture, and this was also inhibited upon MKP-5 overexpression in macrophages within this coculture system (Fig. 5A, B), which further indicated that MKP-5 plays a role in blocking macrophage infiltration during obesity.

To continue assessing the importance of MKP-5 in macrophage-mediated inflammatory response, Raw-PC and Raw-MKP5 cells were treated using CM from cultured adipocytes treated with PA or not. IL- 6 and TNF- $\alpha$ expression levels were upregulated in response to PA-incubated CM treatment. Notably, these increases in proinflammatory cytokines were suppressed in Raw-MKP5 cells relative to Raw-PC cells (Fig. 5C).

These results thus indicate that overexpression of MKP-5 in macrophages may alleviate the inflammation induced upon interactions between macrophages and adipocytes, and that cytokines secreted by PA-treated adipocytes alone may mediate at least a portion of this effect. 
A

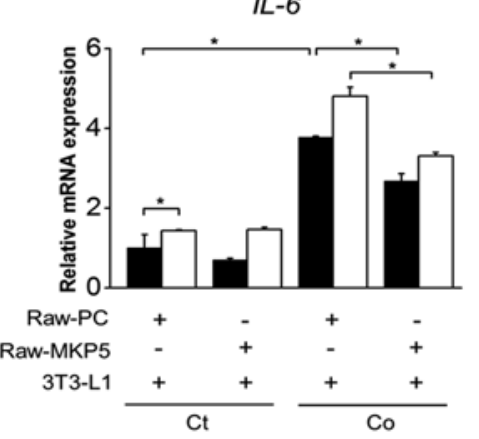

B

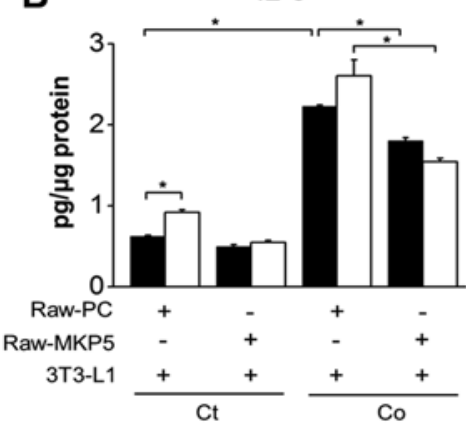

C

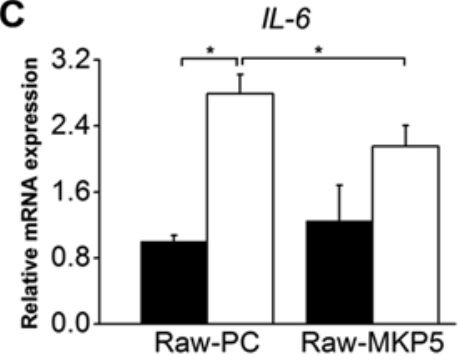

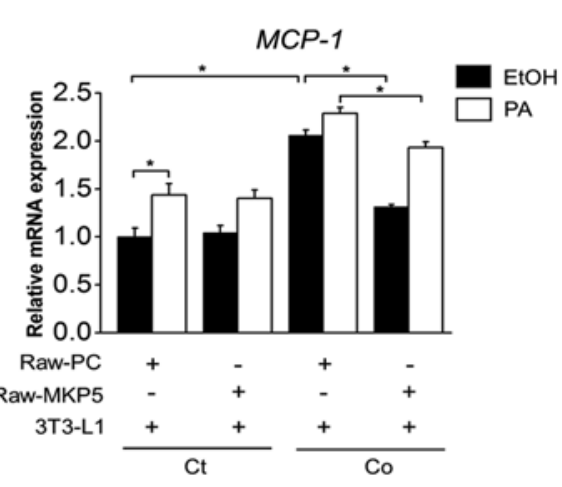

$T N F-\alpha$
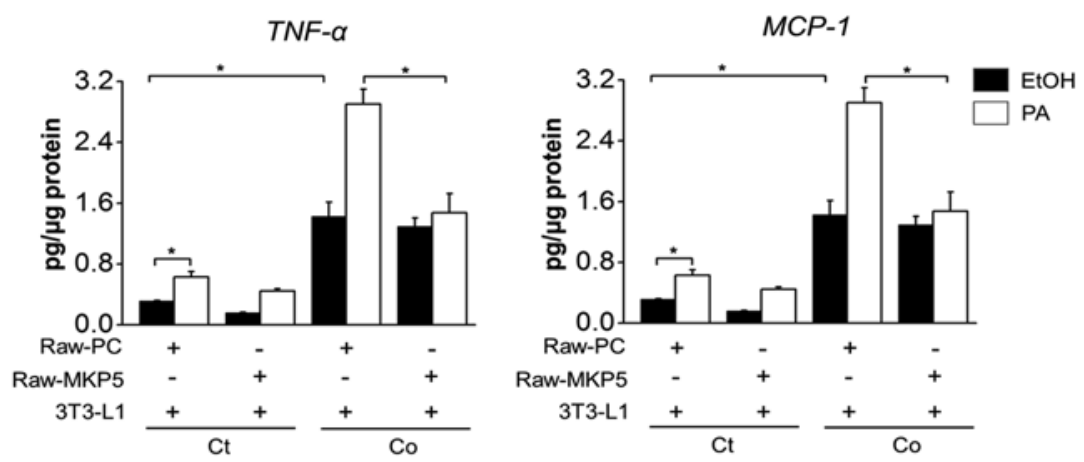

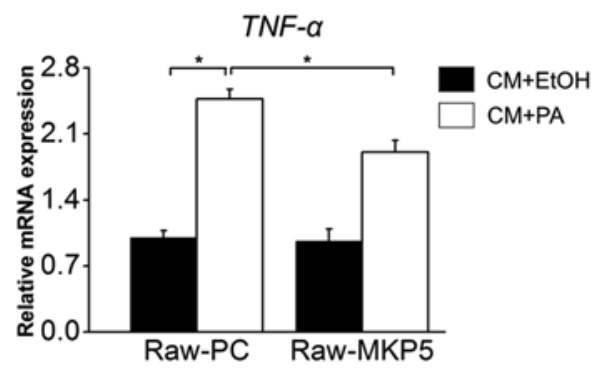

Fig. 5. MKP-5 overexpression in macrophages can alleviate inflammation induced by palmitate (PA) during adipocyte-macrophage coculture. A Raw-PC or Raw-MKP5 and differentiated 3T3-L1 adipocytes were plated either alone or together and stimulated by $500 \mu \mathrm{M}$ PA for $9 \mathrm{~h}$, after which IL- 6 and MCP-1 levels were assessed via quantitative real-time (qRT)-PCR. B The supernatants from the control (Ct) and coculture (Co) groups were collected, and IL-6, TNF- $\alpha$, and MCP-1 levels were measured via ELISA. C Differentiated 3T3-L1 adipocytes were stimulated with PA for $9 \mathrm{~h}$, and then supernatants were collected. Supernatants from unstimulated cells were also collected as a control. Raw-PC and Raw-MKP5 cells were incubated with conditioned medium (CM) collected from 3T3-L1 adipocytes, and IL- 6 and TNF- $\alpha$ levels were measured by qRT-PCR. Results are means \pm SEM of triplicate experiments. EtOH, ethyl alcohol. ${ }^{*} p<0.05$.

\section{MKP-5 Modulates P38, JNK, and ERK Activation in Macrophages in Response to PA}

To examine whether MKP-5, as a dual-specificity phosphatase known to regulate MAPKs, regulates macrophage inflammation via MAPK pathways, we assayed the activation of P38, JNK, and ERK upon PA exposure. We found that P38 and JNK were activated by PA after $0.5 \mathrm{~h}$, and JNK activation increased in a time-dependent manner, with P38 phosphorylation peaked at $6 \mathrm{~h}$, after which it decreased, whereas ERK activation occurred by $6 \mathrm{~h}$ after PA treatment (Fig. 6A). Importantly, MKP-5 overexpression markedly inhibited P38, JNK, and 
A

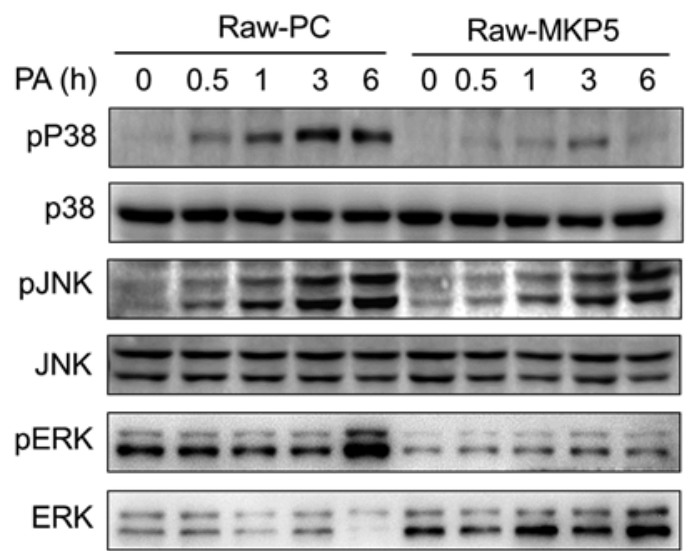

B
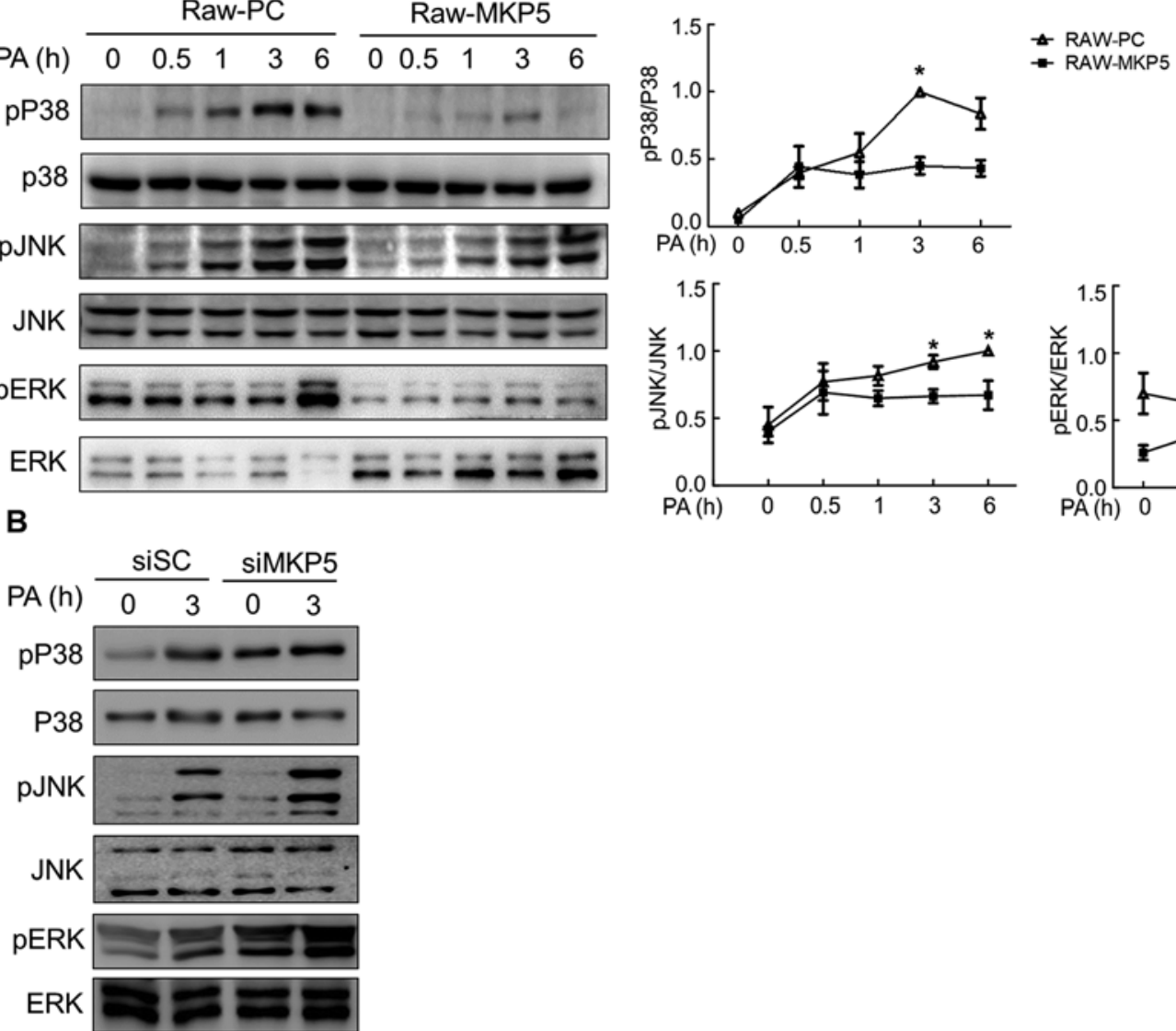

Fig. 6. MKP-5 overexpression inhibits MAPK pathway activation upon palmitate (PA) stimulation. A Raw-PC and Raw-MKP5 cells were treated using $500 \mu \mathrm{M}$ PA, and then JNK, P38, and ERK levels were detected by Western blotting. Protein band intensity was quantified using ImageJ, and data are means \pm SEM of triplicate experiments normalized to total P38, JNK, or ERK. B siRNA-transfected Raw264.7 cells were treated with 500 $\mu \mathrm{M}$ PA for $3 \mathrm{~h}$, and then levels of JNK, P38, and ERK were assessed by Western blotting. * $p<0.05$.

ERK phosphorylation upon PA treatment (Fig. 6A). This inhibitory effect was evident until $6 \mathrm{~h}$ after PA stimulation (Fig. 6A). In contrast, basal P38, JNK, and ERK activation was enhanced after MKP-5 silencing, and their activation was clearly increased after PA treatment for $3 \mathrm{~h}$ (Fig. 6B). Thus, P38, JNK, and ERK are substrates of MKP-5 in macrophages, and may therefore function as key mediators of downstream signaling processes.

MKP-5 Regulates PA-Triggered Macrophage Inflammation through the P38, JNK, and ERK MAPK Pathways

Many previous studies have highlighted a clear role for P38, JNK, and ERK in the context of inflammation. To investigate whether MKP-5 regulates inflammation via the P38, JNK, or ERK pathway, we treated Raw264.7 cells with SB 203580, SP 600125, or U0126, inhibiting P38, JNK, and ERK, respectively, after transfection with control or MKP-5-specific siRNA, and the cells were then treated with PA. The phosphorylation of P38, JNK, and ERK was then assessed by Western blotting. All three inhibitors suppressed activation of the corresponding 


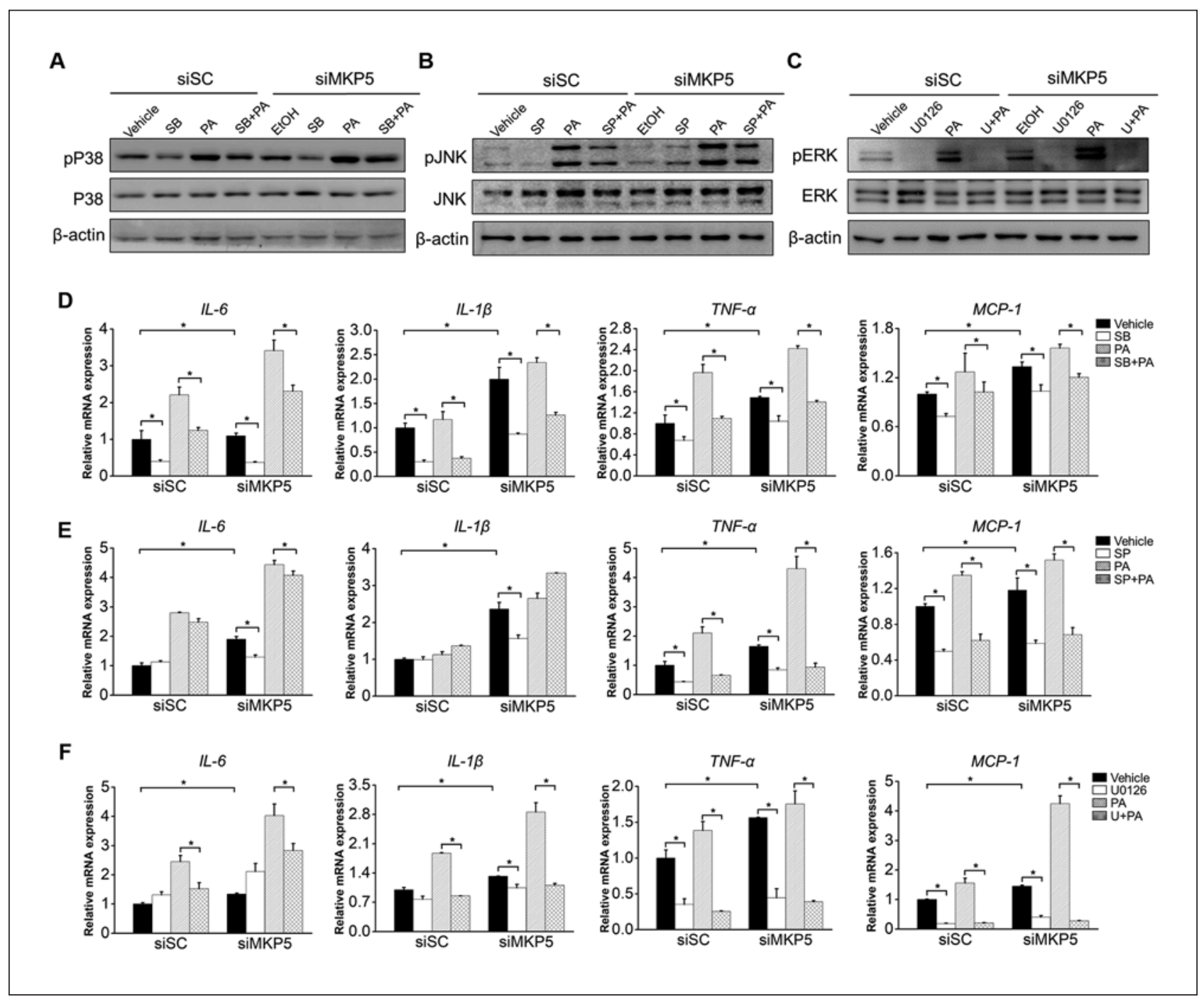

Fig. 7. MKP-5 mediates macrophage inflammation in response to palmitate (PA) by regulating the MAPK pathway. MKP-5 siRNA (siMKP5) or scramble siRNA (SiSC) was used to transfect Raw264.7 cells, which were pretreated with SB 203580 (SB), SP 600125 (SP, or U0126 for $1 \mathrm{~h}$, and were then exposed to $500 \mu \mathrm{M}$ PA for $3 \mathrm{~h}$. A-C Activation of P38, JNK, and ERK was measured by Western blotting. D-F IL-6, IL-1 $\beta$, TNF- $\alpha$, and MCP-1 were quantified by quantitative real-time PCR analysis after treatment with PA and SB 203580 (D), SP 600125 (E), or U0126 (F). Results are means \pm SEM of triplicate experiments. EtOH, ethyl alcohol. * $p<0.05$.

MAPK upon PA treatment; even though activation was enhanced in MKP-5-silenced cells, the inhibitory effect remained apparent (Fig. 7A-C).

The expression levels of the proinflammatory cytokines and the chemokine were significantly increased in MKP-5-silenced cells, and inhibition of the three MAPKs significantly decreased the PA-dependent upregulation of IL-6 and TNF- $\alpha$ (Fig. 7D-F). While the JNK inhibitor did not affect IL-1 $\beta$ expression after PA treatment (Fig. 7E), the inhibitors of P38 and ERK did so (Fig. 7D, F). Notably, this inhibitory effect was significant after MKP-5 knockdown.

These results illustrated that the anti-inflammatory role of MKP-5 was mediated, at least in part, via the inactivation of P38, JNK, and ERK. MCP-1 expression was also downregulated in MKP-5 knockdown cells treated with MAPK inhibitors (Fig. 7D-F), indicating that P38, JNK, and ERK at least in part mediated MKP-5-dependent effects on macrophage infiltration. 
Lu et al.: MKP-5 Relieves PA or Obesity-Induced Inflammation

\section{Discussion}

Obesity is a serious threat to human health, with a close association with chronic lowgrade inflammation that contributes to metabolic disorder. Obese adipose tissue typically exhibits dysfunction, showing significant macrophage infiltration. Adipose tissue macrophages are the primary proinflammatory cytokine-producing cells, secreting TNF- $\alpha$ and IL- 6 in these contexts [5]. In this study, we observed a higher MKP-5 expression in WAT from HFD mice as compared to that from lean mice, which primarily resulted from increased MKP-5 expression in SVCs. Proinflammatory cytokine expression varied in a manner consistent with variations in MKP-5 expression. Therefore, we speculated that MKP-5 might be associated with obesity-induced adipose tissue inflammation, which we ultimately found to be true in vitro and to some extent in vivo. MKP-5 overexpression in macrophages was associated with upregulation of M2 markers and inhibition of M1 marker expression in M1 or M2 polarizing conditions, which indicated a potential role for MKP-5 in promoting M1-to-M2 macrophage cross-polarization. MKP-5 overexpression in Raw264.7 cells also improved the inflammation triggered by PA, as confirmed in MKP-5-overexpressing or -knockdown Raw264.7 cells. These results suggested an inhibitory role for MKP-5 in PA-induced inflammation in vitro. The obesity-induced expression of inflammatory cytokines in SVCs from HFD mice was also suppressed by an adenovirus expressing MKP-5, further confirming that MKP-5 overexpression in SVCs could relieve inflammation in obese adipose tissue. Increasing MKP-5 expression may be a stress response to PA or obesity, aimed at terminating the inflammatory response, although more research is needed on this topic.

In obesity, adipocytes regulate the secretion of IL- 6 and TNF- $\alpha$, as well as of the chemokine MCP-1, to mediate low-grade inflammation. These proinflammatory cytokines stimulate macrophage activation and adipose infiltration [30]. Adipocyte hypertrophy and localized hypoxia further contribute to macrophage recruitment [4]. Therefore, adipocyte-macrophage interactions are crucial to adipose tissue inflammation in response to obesity. Interactions between adipocytes and macrophages are reported to result in IL-6 and MCP-1 secretion [31]. Adipocytes produce FFAs to stimulate macrophage TNF- $\alpha$ secretion, which in turn increases lipolysis and the release of FFAs from adipocytes [12]. Interestingly, TNF- $\alpha$ may increase the expression of MCP-1 via the same paracrine signaling, in turn driving macrophage infiltration [32]. As we found, MKP-5 overexpression in mouse macrophages inhibited the expression of IL- 6 and MCP-1, and further suppressed IL-6, MCP-1, and TNF- $\alpha$ secretion upon adipocytemacrophage coculture. Adipocyte-CM induced a high expression of IL- 6 and TNF- $\alpha$ in macrophages, and this was also suppressed by MKP-5. Therefore, MKP-5 overexpression in macrophages can improve the inflammatory response triggered by adipocyte-macrophage crosstalk, further confirming the critical role of MKP-5 in obesity-induced adipose tissue inflammation.

As a negative regulator of MAPKs, MKP-5 is known to regulate inflammation by mediating inactivation of the MAPKs P38, JNK, and ERK. Most of the previous studies suggested that MKP-5 can inactivate P38 and JNK but not ERK [33], and that MKP-5 preferentially targets P38 and JNK as substrates rather than ERK in vitro [34], although one study suggested that MKP substrate specificities in vivo may differ from their in vitro specificities [35]. Interestingly, we found that MKP-5 was able to inactivate P38, JNK, and ERK, especially in a highly active state upon PA stimulation, indicating that ERK was also an MKP-5 substrate, in addition to P38 and JNK, in Raw264.7 cells. Furthermore, inhibitors of P38, JNK, and ERK can decrease the elevated expression of inflammatory mediators triggered by MKP-5 knockdown regardless of the PA stimulation status, which suggests that MPK5 regulates inflammation via the P38, JNK, and ERK MAPK pathways.

In summary, MKP-5 is an inflammatory inhibitor involved in obesity-induced adipose tissue inflammation and PA-triggered macrophage inflammation. We found that MKP-5 
Lu et al.: MKP-5 Relieves PA or Obesity-Induced Inflammation

promoted macrophages to switch from the M1 to the M2 phenotype, and that the MAPKs P38, JNK, and ERK were mediators via which MKP-5 functioned as an inflammatory inhibitor. This study raises the possibility that MKP-5 may be developed into a potential therapeutic target for obesity-related diseases, including T2DM and IR.

\section{Statement of Ethics}

All experiments conformed to the regulations on the management of experimental animals at Jilin University, with ethical review permission.

\section{Disclosure Statement}

The authors declare no potential conflicts of interest.

\section{Funding Sources}

The authors are grateful for support from the National Natural Science Foundation of China (Grant/Award No. 81500635); the Department of Science and Technology of Jilin Province (Grant/Award No. 20190201151JC); and a Jilin Province-Jilin University Joint Grant (Grant/Award No. SXGJSF2017-1).

\section{Author Contributions}

Y.L. contributed to most of the experiments, analysis of the data, and writing of the manuscript. J.M., P.J., and W.Y. contributed to the design of the study and critical review of the manuscript. J.Z. and Z.S. contributed to data acquisition. Q.Z. and C.Z. contributed to cell culture and Western blotting. X.L., W.T., and W.W. contributed to the animal experiments and real-time PCR assessments.

\section{References}

1 Riddy DM, Delerive P, Summers RJ, Sexton PM, Langmead CJ. G protein-coupled receptors targeting insulin resistance, obesity, and type 2 diabetes mellitus. Pharmacol Rev. 2018 Jan;70(1):39-67.

2 Kusminski CM, Bickel PE, Scherer PE. Targeting adipose tissue in the treatment of obesity-associated diabetes. Nat Rev Drug Discov. 2016 Sep;15(9):639-60.

3 Ralston JC, Lyons CL, Kennedy EB, Kirwan AM, Roche HM. Fatty acids and NLRP3 inflammasome-mediated inflammation in metabolic tissues. Annu Rev Nutr. 2017 Aug;37(1):77-102.

4 Xu H, Barnes GT, Yang Q, Tan G, Yang D, Chou CJ, et al. Chronic inflammation in fat plays a crucial role in the development of obesity-related insulin resistance. J Clin Invest. 2003 Dec;112(12):1821-30.

5 Weisberg SP, McCann D, Desai M, Rosenbaum M, Leibel RL, Ferrante AW Jr. Obesity is associated with macrophage accumulation in adipose tissue. J Clin Invest. 2003 Dec;112(12):1796-808.

6 Grimble RF. Inflammatory status and insulin resistance. Curr Opin Clin Nutr Metab Care. 2002 Sep;5(5):551-9.

7 Rull A, Camps J, Alonso-Villaverde C, Joven J. Insulin resistance, inflammation, and obesity: role of monocyte chemoattractant protein-1 (or CCL2) in the regulation of metabolism. Mediators Inflamm. 2010;2010: 326580.

8 Fujisaka S, Usui I, Bukhari A, Ikutani M, Oya T, Kanatani Y, et al. Regulatory mechanisms for adipose tissue M1 and M2 macrophages in diet-induced obese mice. Diabetes. 2009 Nov;58(11):2574-82.

9 Lumeng CN, Bodzin JL, Saltiel AR. Obesity induces a phenotypic switch in adipose tissue macrophage polarization. J Clin Invest. 2007 Jan;117(1):175-84.

10 McNelis JC, Olefsky JM. Macrophages, immunity, and metabolic disease. Immunity. 2014 Jul;41(1):36-48. 
Lu et al.: MKP-5 Relieves PA or Obesity-Induced Inflammation

11 Prieur X, Mok CY, Velagapudi VR, Núñez V, Fuentes L, Montaner D, et al. Differential lipid partitioning between adipocytes and tissue macrophages modulates macrophage lipotoxicity and M2/M1 polarization in obese mice. Diabetes. 2011 Mar;60(3):797-809.

12 Suganami T, Nishida J, Ogawa Y. A paracrine loop between adipocytes and macrophages aggravates inflammatory changes: role of free fatty acids and tumor necrosis factor alpha. Arterioscler Thromb Vasc Biol. 2005 Oct;25(10):2062-8.

13 Nguyen MT, Favelyukis S, Nguyen AK, Reichart D, Scott PA, Jenn A, et al. A subpopulation of macrophages infiltrates hypertrophic adipose tissue and is activated by free fatty acids via Toll-like receptors 2 and 4 and JNKdependent pathways. J Biol Chem. 2007 Nov;282(48):35279-92.

14 Fujishiro M, Gotoh Y, Katagiri H, Sakoda H, Ogihara T, Anai M, et al. Three mitogen-activated protein kinases inhibit insulin signaling by different mechanisms in 3T3-L1 adipocytes. Mol Endocrinol. 2003 Mar;17(3): 487-97.

15 Gehart H, Kumpf S, Ittner A, Ricci R. MAPK signalling in cellular metabolism: stress or wellness? EMBO Rep. 2010 Nov;11(11):834-40.

16 Nandipati KC, Subramanian S, Agrawal DK. Protein kinases: mechanisms and downstream targets in inflammation-mediated obesity and insulin resistance. Mol Cell Biochem. 2017 Feb;426(1-2):27-45.

17 Zhang Y, Blattman JN, Kennedy NJ, Duong J, Nguyen T, Wang Y, et al. Regulation of innate and adaptive immune responses by MAP kinase phosphatase 5. Nature. 2004 Aug;430(7001):793-7.

18 Zhang Y, Nguyen T, Tang P, Kennedy NJ, Jiao H, Zhang M, et al. Regulation of Adipose Tissue Inflammation and Insulin Resistance by MAPK Phosphatase 5. J Biol Chem. 2015 Jun;290(24):14875-83.

19 Nonn L, Peng L, Feldman D, Peehl DM. Inhibition of p38 by vitamin D reduces interleukin-6 production in normal prostate cells via mitogen-activated protein kinase phosphatase 5: implications for prostate cancer prevention by vitamin D. Cancer Res. 2006 Apr;66(8):4516-24.

20 Wu J, Mei C, Vlassara H, Striker GE, Zheng F. Oxidative stress-induced JNK activation contributes to proinflammatory phenotype of aging diabetic mesangial cells. Am J Physiol Renal Physiol. 2009 Dec;297(6):F1622-31.

21 Choi HR, Kim WK, Kim EY, Han BS, Min JK, Chi SW, et al. Dual-specificity phosphatase 10 controls brown adipocyte differentiation by modulating the phosphorylation of p38 mitogen-activated protein kinase. PLoS One. 2013 Aug;8(8):e72340.

22 Jiao P, Chen Q, Shah S, Du J, Tao B, Tzameli I, et al. Obesity-related upregulation of monocyte chemotactic factors in adipocytes: involvement of nuclear factor-kappaB and c-Jun NH2-terminal kinase pathways. Diabetes. 2009 Jan;58(1):104-15.

23 Sakamoto Y, Kanatsu J, Toh M, Naka A, Kondo K, Iida K. The dietary isoflavone daidzein reduces expression of pro-inflammatory genes through PPAR $\alpha / \gamma$ and JNK pathways in adipocyte and macrophage co-cultures. PLoS One. 2016 Feb;11(2):e0149676.

24 Lewandowska E, Zieliński A. White adipose tissue dysfunction observed in obesity [in Polish]. Pol Merkur Lekarski. 2016 May;40(239):333-6.

25 Guzik TJ, Skiba DS, Touyz RM, Harrison DG. The role of infiltrating immune cells in dysfunctional adipose tissue. Cardiovasc Res. 2017 Jul;113(9):1009-23.

26 Zeyda M, Stulnig TM. Adipose tissue macrophages. Immunol Lett. 2007 Oct;112(2):61-7.

27 Locati M, Mantovani A, Sica A. Macrophage activation and polarization as an adaptive component of innate immunity. Adv Immunol. 2013;120:163-84.

28 Zhuge F, Ni Y, Nagashimada M, Nagata N, Xu L, Mukaida N, et al. DPP-4 inhibition by linagliptin attenuates obesity-related inflammation and insulin resistance by regulating M1/M2 macrophage polarization. Diabetes. 2016 Oct; 65(10):2966-79.

29 Maurizi G, Della Guardia L, Maurizi A, Poloni A. Adipocytes properties and crosstalk with immune system in obesity-related inflammation. J Cell Physiol. 2018 Jan;233(1):88-97.

30 Shoelson SE, Herrero L, Naaz A. Obesity, inflammation, and insulin resistance. Gastroenterology. 2007 May; 132(6):2169-80.

31 Sárvári AK, Doan-Xuan QM, Bacsó Z, Csomós I, Balajthy Z, Fésüs L. Interaction of differentiated human adipocytes with macrophages leads to trogocytosis and selective IL-6 secretion. Cell Death Dis. 2015 Jan;6(1):e1613.

32 Sartipy P, Loskutoff DJ. Monocyte chemoattractant protein 1 in obesity and insulin resistance. Proc Natl Acad Sci USA. 2003 Jun;100(12):7265-70.

33 Min K, Lawan A, Bennett AM. Loss of MKP-5 promotes myofiber survival by activating STAT3/Bcl-2 signaling during regenerative myogenesis. Skelet Muscle. 2017 0ct;7(1):21.

34 Theodosiou A, Smith A, Gillieron C, Arkinstall S, Ashworth A. MKP5, a new member of the MAP kinase phosphatase family, which selectively dephosphorylates stress-activated kinases. Oncogene. 1999 Nov;18(50): 6981-8.

35 Zhang Y, Dong C. Regulatory mechanisms of mitogen-activated kinase signaling. Cell Mol Life Sci. 2007 Nov; 64(21):2771-89. 